\title{
Pulsed Laser Melting Effects on Single Crystal Gallium Phosphide
}

\author{
D. Pastor, J. Olea, M. Toledano-Luque, I. Mártil, and G. González-Díaz \\ Dpto. de Física Aplicada III (Electricidad y Electrónica), Facultad de Ciencias Físicas, \\ Universidad Complutense de Madrid, Madrid E-28040, Spain \\ J. Ibáñez, R. Cuscó and L. Artús \\ Institut Jaume Almera, Consejo Superior de Investigaciones Científicas (CSIC), \\ Lluís Solé i Sabarís s/n, 08028 Barcelona, Spain
}

\begin{abstract}
We have investigated the pulse laser melting (PLM) effects on single crystal GaP. The samples have been studied by means of Raman spectroscopy, glancing incidence $X$-ray diffraction (GIRXD), van der Pauw and Hall effect measurements. After PLM process, the Raman spectra of samples annealed with the highest energy density show a forbidden TO vibrational mode of GaP. This suggests the formation of crystalline domains with a different orientation in the GaP PLM region regarding to the GaP unannealed region. This behavior has been corroborated by glancing incidence $x$-ray diffraction measurements. A slightly increase in the sheet resistivity and a suppression of the mobility in PLM samples have been observed in all the measured temperature range. Such annealing effects are a cause of great concern for intermediate band (IB) materials formation where PLM processes are required first, to recovery the lattice crystallinity after high dose ion implantation processes and second, to avoid impurities outdiffusion when the solid solubility limit is exceeded.
\end{abstract}

\section{INTRODUCTION}

During last years many efforts have been carried out to increase the efficiency in solar cells based on single crystal semiconductors. In 1997, a theoretical paper showed that the introduction in a host semiconductor of a so called intermediate band (IB) could enhance drastically the efficiency in single junction solar cells [1]. Since then, further papers describing the properties of such IB have been reported $[2,3]$. The main feature of an IB material is a new band localized between the conventional valence band (VB) and the conduction band $(\mathrm{CB})$ of the semiconductor. The advantage of this new band is that electrons of energy below the band gap can be promoted from VB into CB by means of two sub-band gap photon absorption of lower energy using the IB like an intermediary.

The IB obtaining has been theoretically proposed and experimentally certified in quantum dots (QD) based devices [4]. Actually many efforts have been realized to improve this QD materials that led to obtain an optimal efficiency [5].

Regarding single crystal semiconductors, the solar energy materials research group of the Lawrence Berkeley National Laboratory has showed the formation of an IB in different diluted semiconductors such as GaInNAs [6], ZnMnOTe [7] and GaNAsP [8].

Theoretical calculations point to $\mathrm{GaP}$ as one of the most promising materials for the formation of an IB by means of the introduction of deep levels in concentrations above the Mott limit [2], that is usually well above the solubility limit [9-11].

In most cases, the high concentration of impurities required for the IB formation exceeds the solid solubility limit of impurities in the bulk semiconductor. Whereas most doping techniques do not allow reaching the impurity concentration needed for the IB formation, ion implantation is a powerful technique that permits the controlled introduction of a high impurity concentration into the bulk semiconductor above the mentioned solubility limit [12]. However, the ion implantation damages the crystalline lattice of the host semiconductor, thus thermal annealing treatments are required in order to recover the crystallinity. Given that annealing processes under equilibrium conditions could produce impurity out-diffusion from the semiconductor, non equilibrium annealing processes like pulsed laser melting (PLM) are necessary. In this way, the PLM process is revealed like a technological key to obtain an IB in bulk semiconductors. The effect of the PLM process on III-V semiconductors has been reported $[8,13,14]$. As far we are concerned no such study has been carried out on GaP.

In this work, we present an optical, structural and electrical study of the PLM effects on GaP physical properties. We observe clearly forbidden vibrational modes by means of Raman Spectroscopy. We have carried out also Glancing Incidence X-ray diffraction (GIRXD) corroborating this behavior. We have investigated the electrical properties of these samples showing an increase of the sheet resistivity and a suppression of the mobility.

\section{EXPERIMENTAL}

The samples used in this study were undoped (LEC) (100) $\mathrm{GaP}$ wafers with a thickness of $500 \mu \mathrm{m}$ and a resistivity at room temperature of $\rho=0.3 \Omega . \mathrm{cm}$. The samples were annealed at J.P. Sercel Associates, Inc. (New Hampshire, USA) with a $\operatorname{KrF}$ excimer laser $(\lambda=248 \mathrm{~nm})$ at energy densities from 0.2 to $1.2 \mathrm{~J} / \mathrm{cm}^{2}$, with a single $20 \mathrm{~ns}$ pulse. For this wavelenght the 
annealed region depth in $\mathrm{GaP}$ is about of tens of nanometers [12].

The Raman spectra were carried out with a Jobin-Yvon T64000 triple-grating spectrometer equiped with a coupled charge detector cooled with liquid nitrogen. All the spectra have been acquired in unpolarized backscattering configuration on a (100) face. The $325 \mathrm{~nm}$ line of an He-Cd laser was used for excitation. For this wavelenght, taking into account the corresponding absorption coefficient of GaP [15], we estimate that the Raman signal originates from a surface layer thickness of about $10 \mathrm{~nm}$, which is smaller than the depth of the PLM region.

X-ray difracction (XRD) was performed to study the crystallinity of the PLM region using a analytical X'Pert PRO MRD diffractometer with $\mathrm{Cu} \mathrm{K}_{\alpha}$ radiation in glancing incidence configuration (GIXRD). This technique provides depth-resolved information about the crystal structure by means of the incidence angle of the $\mathrm{x}$-ray source and permits us to probe a depth of tens of nanometers [16], about the same thickness order of the PLM layer. Previous to GIRXD measurements, $\theta-2 \theta$ scans were performed to obtain the same orientation sample reference to asses the same sample measurements conditions.

$\mathrm{GaP}$ samples were electrically characterized by Hall effect measurements in the van der Pauw configuration at variable temperature using a Keithley SCS 4200 model, setting the sample inside a homemade variable temperature sample holder attached to a vacuum pump to avoid moisture condensation. Temperature varied between 90 and $380 \mathrm{~K}$. A Kepco BOP 50$20 \mathrm{MG}$ bipolar power supply fed the electro-magnet, reducing the thermo-galvanic-magnetic effects by changing the magnetic flux direction.

The GaP metal contacts for electrical measurements were made in a Pfeiffer Classic 250 evaporator model. A multilayer metalization scheme: $\mathrm{Ni}(50 \mathrm{~nm})$ layer, $\mathrm{Au} / \mathrm{Ge}(88: 12$ in weight) layer $(200 \mathrm{~nm})$ were carried out in order to obtain ohmic contacts in the n-type GaP substrate. Finally, whereas for the PLM samples no additional step has been required to achieve ohmic contacs, for the unannealed $\mathrm{GaP}$ reference sample, a rapid thermal annealing (RTA) at $500{ }^{\circ} \mathrm{C}$ for $30 \mathrm{~s}$ has been necessary.

\section{RESULTS}

Fig. 1 shows the unpolarized Raman spectra of $\mathrm{GaP}$ samples annealed at different PLM energy densities $(0.2,0.7$ and 1.2 $\mathrm{J} / \mathrm{cm}^{2}$ ). We also show the spectrum of the unannealed GaP substrate. According to the selection rules for the zinc-blende structure only the longitudinal optical (LO) mode is allowed in the backscattering configuration from the (100) face. The Raman spectrum of the as-grown sample is dominated by the peak at $405 \mathrm{~cm}^{-1}$ which is assigned to the LO mode [17]. Whereas the LO mode of the sample annealed at $0.2 \mathrm{~J} / \mathrm{cm}^{2}$ shows only a slight intensity decrease, the Raman spectra of the samples annealed at energy densities higher than $0.7 \mathrm{~J} / \mathrm{cm}^{2}$ are substantially altered, as it displays a strong transversal optical (TO) peak at $367 \mathrm{~cm}^{-1}$ which is forbidden in this scattering geometry. The observation of a forbidden TO mode in the Raman spectra suggests that the PLM process gives rise

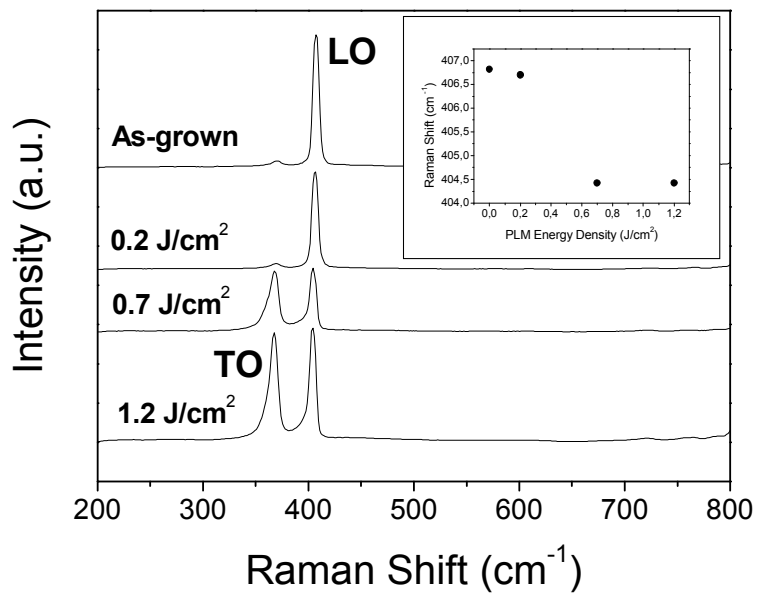

Fig. 1. Room-temperature Raman spectra of a LEC-grown GaP annealed at different energy densities.

to the formation of a $\mathrm{GaP}$ layer with disoriented domains, typical of polycrystalline material. A similar behavior has been observed previously in ion implanted InP epilayers, after annealing [13].

Whereas there is no appreciable differences in the LO peak width between the unannealed sample and the sample annealed at $0.2 \mathrm{~J} / \mathrm{cm}^{2}$, the LO peak of the sample annealed at $0.7 \mathrm{~J} / \mathrm{cm}^{2}$ is broadened to lower frequencies. This increase in the width of the LO peak together with the presence of a forbidden TO peak, broadened both to downward frequency indicates a reduction in the crystalline quality, probably induced by the presence of defects of the boundary domains of the different orientations. This poor lattice crystallinity suggests that the decrease of the LO-mode intensity of this sample can not only explained in terms of a decrease in the scattering volume of (100) oriented regions and the consequent increase of the TO mode intensity contributions of the disoriented regions.

Since in the spectrum of the sample annealed at $0.2 \mathrm{~J} / \mathrm{cm}^{2}$ there is only slightly decreased in the intensity of the LO mode, and there is not an appreciable broad peak differences, we can conclude that the PLM process at this energy does not produce a substantial structural change.

Conversely, the sample annealed at $0.7 \mathrm{~J} / \mathrm{cm}^{2}$ presented a forbidden peak which is likely due to disoriented domains. Finally, when the PLM energy density was increased up to 1.2 $\mathrm{J} / \mathrm{cm}^{2}$, the TO and LO vibrational modes still persisted but they were slightly narrowed and more intense suggesting a certain crystalline improvement relative to the sample annealed at $0.7 \mathrm{~J} / \mathrm{cm}^{2}$.

In the inset of Fig. 1, another key parameter is plotted: the frequency of the LO mode for the different annealed GaP samples. It is worth highlighting that the samples annealed with an energy density of 0.7 and $1.2 \mathrm{~J} / \mathrm{cm}^{2}$ present a shift in the peak of the LO mode to downward frequency of about 3 $\mathrm{cm}^{-1}$. This shift has been observed repetitively in different $\mathrm{GaP}$ samples annealed in the same conditions and is a clearly indicative of strain presence in these samples that could be related with the boundary crystalline domain formation or with 


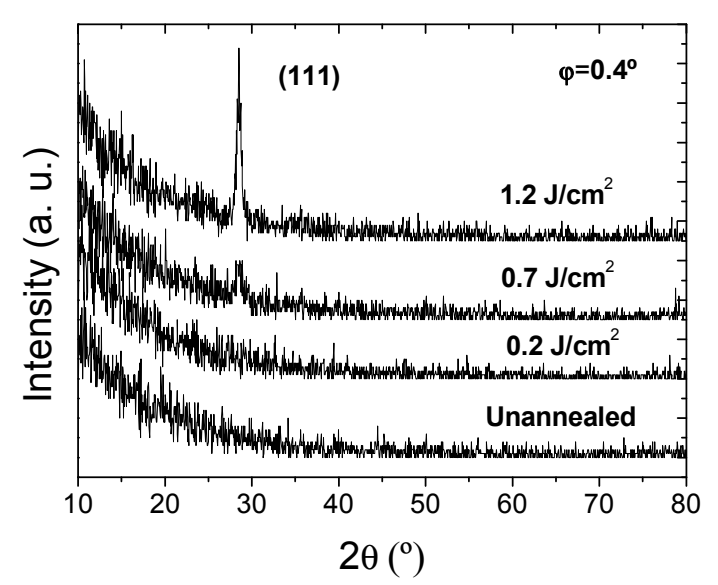

Fig. 2 GIXRD patterns for an unannealed GaP substrate and for GaP samples annealed at $0.2,0.7$ and $1.2 \mathrm{~J} / \mathrm{cm}^{2}$. All these measurements were taken at glancing angle of $\varphi=0.4^{\circ}$.

the presence of defects. The clear structural differences between the sample annealed at the lowest density of energy $\left(0.2 \mathrm{~J} / \mathrm{cm}^{2}\right)$ and the samples annealed at the highest energy densities $\left(0.7\right.$ and $\left.1.2 \mathrm{~J} / \mathrm{cm}^{2}\right)$ seems to point out that the GaP melting has been taken place in these latest. Therefore, laser annealing would produce the $\mathrm{GaP}$ melting in the samples annealed from $0.7 \mathrm{~J} / \mathrm{cm}^{2}$, where the structural changes are observed. The forbidden TO mode feature observation, attributed to the formation of different orientated domains, would be due to a hastied recrystallization as temperature is fast decreased from GaP melt point after PLM. Another explanation could be related with a superficial $\mathrm{GaP}$ decomposition, where a high density of point defects related to $\mathrm{P}$ vacancies could be created and could generate a $\mathrm{GaP}$ regrowth layer with disorientated domains.

The crystalline structure of the samples has been also characterized by means of GIXRD. Fig. 2 shows the GIXRD measurements acquired with a $\varphi=0.4^{\circ}$ glancing angle for the laser annealed layers at $0.2,0.7$ and $1.2 \mathrm{~J} / \mathrm{cm}^{2}$. Unannealed $\mathrm{GaP}$ sample measurement has been also included for comparative purposes. All these measurements have been carried out at the same glancing incidence angle and the same sample orientation. No peaks have been observed in the unannealed GaP diffraction pattern neither in the sample annealed with the lowest density energy $\left(0.2 \mathrm{~J} / \mathrm{cm}^{2}\right)$.

This result shows that the annealing at $0.2 \mathrm{~J} / \mathrm{cm}^{2}$ does not produce any significant effect in the GaP lattice structure. However, the XRD patterns of the samples annealed at 0.7 and $1.2 \mathrm{~J} / \mathrm{cm}^{2}$ present a new peak attributed to the (110) reflection of GaP. This peak is due to different orientated domains from the $\mathrm{GaP}$ unannealed region. The higher intensity and narrower width of this peak in the sample annealed at the highest density energy $\left(1.2 \mathrm{~J} / \mathrm{cm}^{2}\right)$ with regard to the sample annealed at 0.7 $\mathrm{J} / \mathrm{cm}^{2}$, could indicate a decrease in effective area of defects, thus an increase in the size of different disorientated crystalline domains. All these results are in full agreement with the results obtained by Raman spectroscopy.

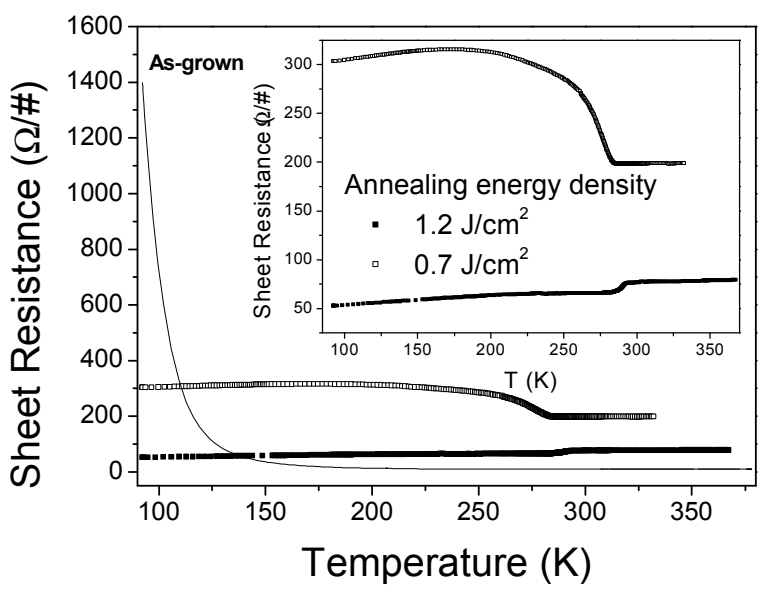

Fig. 3. Sheet resistance as a function of measured temperature of GaP samples at different PLM energy densities: 0.7 and $1.2 \mathrm{~J} / \mathrm{cm}^{2}$ and as-grown $\mathrm{GaP}$.

Fig. 3 shows the sheet resistance as a function of temperature for PLM GaP at 0.7 and $1.2 \mathrm{~J} / \mathrm{cm}^{2}$. The sheet resistance of asgrown $\mathrm{GaP}$ substrate is also plotted for comparison purposes. As can be seen, the sheet resistance of the PLM samples is higher than the sheet resistance of the unannealed GaP. This effect increases as the PLM energy density decreases. These results can be explained in terms of the polycrystallinity of the annealed sample and of the poorer crystallinity in the case of sample annealed at $0.7 \mathrm{~J} / \mathrm{cm}^{2}$. The sample annealed at 0.2 $\mathrm{J} / \mathrm{cm}^{2}$ has not been possible to measure. This seems to be in agreement with the fact that unannealed sample only has been possible to measure after a RTA at $500{ }^{\circ} \mathrm{C}$ during $30 \mathrm{~s}$, so the annealing at $0.2 \mathrm{~J} / \mathrm{cm}^{2}$ has not introduced a significant change in the crystalline structure.

Differences in the sheet resistance between two different van der Pauw configurations that could not be explained by means of a geometry factor, has been observed in the annealed samples at 0.7 and $1.2 \mathrm{~J} / \mathrm{cm}^{2}$, but not in the unannealed sample. This could reflect a certain crystal anisotropy caused by the disoriented domains formation in the samples were the melt of the annealed layer has been taken place.

Fig. 4 shows the Hall mobility measurements of the unannealed and PLM samples. Whereas the Hall mobility in the unannealed sample has the typical behavior associated with phonon scattering, the Hall mobility in annealed samples is essentially zero. This indicates that the polycrystalline surface layer induced by PLM produces a strong undesirable effect in the $\mathrm{GaP}$ electrical properties.

\section{CONCLUSION}

Summarizing, the observation of forbidden vibrational Raman modes in PLM samples indicates polycrystalline surface layer formation. These additional forbidden peaks are not clearly visible until PLM density energy of $0.7 \mathrm{~J} / \mathrm{cm}^{2}$ is reached, suggesting that is the minimum density energy to produce the $\mathrm{GaP}$ melt. 


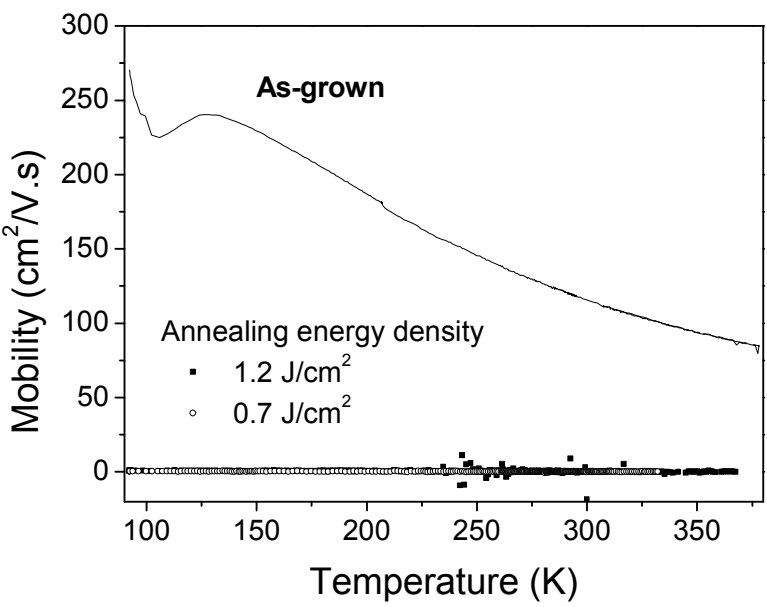

Fig. 4. Hall mobility as a function of measured temperature of GaP layers at different annealing energy densities: 0.7 and $1.2 \mathrm{~J} / \mathrm{cm}^{2}$ and as-grown GaP.

GIRXD measurements, in agreement with the Raman spectroscopy results, show disorientated crystalline domains after annealing at the highest energy densities. Electrical characterization shows an increase of the sheet resistance as annealing density energy density is increased, and a suppression of the mobility in all the annealed samples measured. These effects constitute a handicap for achieving IB formation in GaP by means of ion implantation and subsequent PLM processing, and indicate that further work is still needed.

\section{ACKNOWLEDGMENT}

Authors would like to acknowledge the CAI of X-ray diffraction of the Complutense University of Madrid for GIXRD measurements and the CAI of Técnicas Físicas of the Complutense University of Madrid for ohmic contact evaporation for electric measurements. This work was made possible thanks to the FPI program (Grant No. BES-20057063) and to the contract MAT2007-63617 of the Spanish Ministry of Education and Science. This work was partially supported by the Project NUMANCIA (Grant No. S0505/ENE/000310) funded by the Comunidad de Madrid and by the Project GENESIS-FV (Grant No. CSD2006-00004) funded by the Spanish Consolider National Program.

\section{REFERENCES}

[1] A. Luque, A. Marti, "Increasing the efficiency of ideal solar cells by photon induced transitions at intermediate levels", Physical Review Letters, vol. 78, pp. 5014-5017, February 1997.

[2] A. Luque, A. Marti, E. Antolín, C. Tablero, "Intermediate bands versus levels in non radiative recombination," Physica B, vol. 382, pp.321-327, March 2006.

[3] L. Cuadra, A. Martí, and A. Luque, "Influence of the overlap between the absorption coefficients on the efficiency of the intermediate solar band cell," IEEE Transactions on Electron Devices, vol. 51, pp. 10021007, June 2004.

[4] A. Martí, E. Antolín, C. R. Stanley, C. D. Farmer, N. López, P. Díaz, E.Cánovas, P. G. Linares, and A. Luque "Production of Photocurrent due to Intermediate-to-Conduction-Band Transitions: A Demonstration of a Key Operating Principle of the Intermediate-Band Solar Cell,"
Physical Review Letters, vol. 97, pp. 247701.1- 247701.4, December 2006.

[5] A. Luque, A. Martí, N. López, E. Antolín, E. Cánovas, C. Stanley, C. Farmer, L.J. Caballero, 1. Cuadra and J.L. Balenzategui, "Experimental Analysis of the quasi Fermi level split in quantum dot intermediate-band solar cells," Applied Physics Letters, vol. 87, pp. 0835505.1-0835505.3, August 2005.

[6] W. Shan, W. Walukiewicz, J. W. Ager III, E. E. Haller, J. F. Geisz, B. J. Friedman, J. M. Olson and S. R. Kurtz, "Band Anticrossing in GaInNAs Alloys", Physical Review Letters, vol. 82, pp. 1221-1224, February 1999.

[7] K. M. Yu, W. Walukiewicz, J. Wu, W. Shan, J. W. Beeman, M. A. Scarpulla, O. D. Dubon and P. Becla, "Diluted II-VI Oxide Semiconductors with Multiple Band Gaps," Physical Review Letter, vol. 91, pp. 246403.1-246403.4, December 2003.

[8] K. M. Yu, W. Walukiewicz, J. W. Ager III, D. Bour, R. Farshchi, O. D. Doubon, S. X. Li, D. Sharp and E. E. Haller, "Multiband GaNAsP quaternary alloys," Applied Physics Letters, vol. 88, pp. 092110.1092110-3, December 2006.

[9] P. Wahnón, C. Tablero, "Ab initio electronic structure calculations for metallic intermediate band formation in photovoltaic materials", Physical Review B, vol. 65, pp. 165115.1-165115.10, April 2002.

[10] C. Tablero, P. Wahnón, "Analysis of metallic intermediate-band formation in photovoltaic materials," Applied Physics Letters, vol. 82, pp. 151-153, November 2003.

[11] C. Tablero, "Analysis of the electronic properties of intermediate band materials as function of impurity concentration," Physical Review B, vol. 72, pp. 035213.1-035213.6, July 2005.

[12] J. Olea, M. Toledano-Luque, D. Pastor, G. González-Díaz and I. Mártil, "Titanium doped silicon layers with very high concentration," Journal of Applied Physics, vol. 104, pp. 0165105.1-0165105.3, July 2008.

[13] C. Pizzuto, G. Vitali, G. Zollo, D. Karpuzov, M. Kalitzova, P. van der Heide, G. Scamarcioo, V. Spagnolo, L. Chiavarone, "Structural reordering and electrical activation of ion-implanted GaAs and InP due to layer in a controlled atmosphere," Physical Review B, vol. 59, pp. 2986-2994, January 1999.

[14] K. M. Yu, W. Walukiewicz, J. Wu, W. Shan, and J. W. Beeman, M. A. Scarpulla, O. D. Dubon, M. C. Ridgway, "Mutual passivation of group IV donors and nitrogen in diluted $\mathrm{GaN}_{\mathrm{x}} \mathrm{As}_{1-\mathrm{x}}$ alloys," Applied Physics Letters vol. 83, pp. 2844-2846, October 2003.

[15] D.E. Aspnes D.E. and A. A. Studna, "Dielectric functions and optical parameters of $\mathrm{Si}, \mathrm{Ge}, \mathrm{GaP}, \mathrm{GaAs}, \mathrm{GaSb}, \mathrm{InP}, \mathrm{InAs}$ and $\mathrm{InSb}$ from 1.5 to 6.0 ev.," Physical Review B vol. 27,pp. 985-1009, January 1983.

[16] E.D. Specht, C.J. Sparks, and C.J. Mc Hargue, "Determination of residual stress in Cr-implanted $\mathrm{Al}_{2} \mathrm{O}_{3}$ by glancing angle $\mathrm{x}$-ray diffraction," Applied Physics Letters, vol. 60, pp. 2216-2218, February 1992.

[17] M. V. Hobden, J.P. Rusell, "The Raman Spectrum of Gallium Phosphide," Physics Letters, vol. 13, pp. 39-41, November 1964. 